\title{
Joint predictive value of c'TnI and NT- proBNP on mortality in patients with coronavirus disease 2019: A retrospective research in Wuhan, China
}

\author{
Haoyu Weng ${ }^{1, \#}$, Fan Yang ${ }^{1, \#, ~ L o n g ~ Z h a n g ~}{ }^{1}$, Han Jin ${ }^{1}$, Shengcong Liu ${ }^{1}$, \\ Fangfang Fan ${ }^{1}$, Zhihao Liu ${ }^{1}$, Xizi Zheng ${ }^{2}$, Hongyu Yang ${ }^{2}$, Yuxi Li ${ }^{1}$, Tieci Yi ${ }^{1}$, \\ Haichao $\mathrm{Li}^{3}$, Yan Zhang ${ }^{1}$, Jianping $\mathbf{L i}^{1,4}$ \\ 'Department of Cardiology, Peking University First Hospital, No. 8 Xishiku Street, Xicheng \\ District, Beijing 100034, China; \\ ${ }^{2}$ Department of Nephrology, Peking University First Hospital, No. 8 Xishiku Street, Xicheng District, Beijing \\ 100034, China; \\ ${ }^{3}$ Department of Respiratory and Critical Care Medicine, Peking University First Hospital, No. 8 Xishiku Street, \\ Xicheng District, Beijing 100034, China \\ ${ }^{4}$ Key laboratory of Molecular Cardiology Sciences of the Ministry of Education, Peking University Health \\ Science Center, No.38 Xueyuan Road, Beijing 100191, China
}

"Haoyu Weng and Fan Yang contributed equally to the research as co-first authors.

Address for Correspondence: Yan Zhang, MD, Department of Cardiology, Peking University First Hospital, No. 8 Xishiku Street, Xicheng District, Beijing 100034, China Email: drzhy1108@163.com

Jianping Li, MD, PhD, Department of Cardiology, Peking University First Hospital, Beijing, China

No. 8 Xishiku Street, Xicheng District, Beijing 100034, China

E-mail: lijianping03455@pkufh.com.

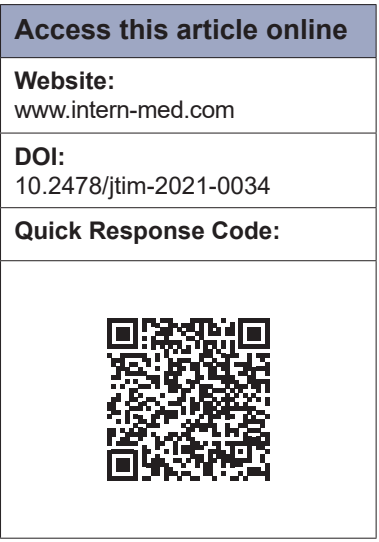

\section{ABSTRACT}

Background and Objectives: The pandemic of coronavirus disease 2019 (COVID-19) remains to be the biggest public threat all over the world. Because of the rapid deterioration in some patients, markers that could predict poor clinical outcomes are urgently required. This study was to evaluate the predictive values of cardiac injury parameters, including cardiac troponin I (cTnl) and N-terminal pro-B-type natriuretic peptide (NT-proBNP) levels, on mortality in COVID-19 patients. Methods: COVID-19 patients in Zhongfaxincheng branch of Tongji Hospital (Wuhan, China) from February 8-28, 2020, were enrolled in this study. We followed up the patients for 30 days after admission. Results: A total of 134 patients were included in the study. Multivariate Cox regression showed that 1) patients with elevated cTnl levels had a higher risk of death (hazard ratio [HR] 7.33, 95\% confidence interval [Cl] 2.56-21.00) than patients with normal cTnl levels; 2) patients with elevated NT-proBNP levels had a higher risk of death (HR 27.88, 95\% Cl 3.55-218.78) than patients with normal NT-proBNP levels; 3 ) patients with both elevated $\mathrm{cTnl}$ and NT-proBNP levels had a significantly higher risk of death (HR 53.87, 95\% Cl 6.31-459.91, $P<0.001$ ) compared to patients without elevated $\mathrm{cTnl}$ or NT-proBNP levels; 4) the progressions of cTnl and NT-proBNP levels were also correlated with death (HR 12.70, 95\% Cl 3.94-40.88, $P<0.001$ and HR 51.09, 95\% Cl 5.82-448.26, $P<$ 0.001). Conclusions: In COVID-19 patients, CTnl and NT-proBNP levels could be monitored to identify patients at a high risk of death in their later course of disease.

Key words: coronavirus disease 2019 , cTnl, NT-proBNP, cardiac injury, predictive

\section{INTRODUCTION}

Since its first outbreak in Wuhan, China, the pandemic of coronavirus disease 2019 (COVID-19) has spread to 215 countries, attached to more than 135 million people, and led to more than 2.9 million deaths. It is still the biggest public health threat all over the world. Compared to previous diseases caused by the coronavirus, such as severe acute respiratory syndrome (SARS) and Middle East respiratory syndrome (MERS), COVID-19 is characterized by a higher infection rate and a lower death rate. ${ }^{[1]}$ However, given the huge number of infected patients, identifying the high-risk patients and giving them more attention is the priority for clinicians to improve the patients' outcomes. 
A big challenge for reducing case fatality rate is the unpredictable progression of the disease. Patients may present as stable in the early stage and then undergo dramatic exacerbation in the later stage or even in the process of recovery. So, identifying individuals' characters that could predict poor outcomes has been the priority for investigators. Several clinical characters, such as old age, concomitant cardiovascular or respiratory diseases, diabetes, and cancer, have been proposed to be related with poor clinical outcomes. ${ }^{[2,3]}$

Laboratory abnormalities, including elevated markers of inflammation, coagulation disorder, and cardiac injury, have also been correlated with disease severity. ${ }^{[2,4]}$ Among the laboratory abnormalities, parameters indicating cardiac injury have aroused most research interests. Cardiac troponin I (cTnI) is a sensitive indicator for cardiomyocyte injury, and it was reported that $20-30 \%$ of patients with COVID-19 had increased cTnI levels, ${ }^{[5]}$ and nonsurvivors or critically ill patients had a higher rate of cTnI elevation than survivors or mild cases. ${ }^{[2,4,6,7]}$ Several studies have shown the association between the cardiac injury markers and worse clinical outcomes, including death. ${ }^{[2,8]} \mathrm{N}$-terminal pro-Btype natriuretic peptide (NT-proBNP) is another parameter indicating cardiac injury, and it has been used as a sensitive indicator of heart function insufficiency. In a recent report of hospitalized Chinese COVID-19 patients, 52\% of nonsurvivors suffered heart failure, compared to $12 \%$ of the survivors. ${ }^{[2]}$ Recent studies have also shown the association of elevated NT-proBNP levels with poor outcomes in COVID-19 patients. $^{\left[{ }^{[]}\right.}$

Though both cTnI and NT-proBNP levels have been reported to be associated with poor clinical outcomes in COVID-19 patients, no study has tried to explore the joint predictive value of the two parameters. Therefore, in this retrospective, single-center study, we investigated the associations of cTnI levels, NT-proBNP levels, as well as their joint effects on mortality in patients with COVID-19. Moreover, we explored the effect of the progression of cTnI and NT-proBNP levels on mortality in our patients.

\section{METHODS}

\section{Study participants}

In early February 2020, a medical team from Peking University First Hospital, Peking University People's Hospital, and Peking University Third Hospital was sent to Wuhan to support local treatment of COVID-19 patients. They were assigned to treat severe and critically ill patients in Zhongfaxincheng branch of Tongji Hospital (Wuhan, China). From February 8-28, 2020, 138 patients were transferred to their wards. All patients were diagnosed with COVID-19 in accordance with the criteria of WHO interim
138 patients with confirmed COVID-19

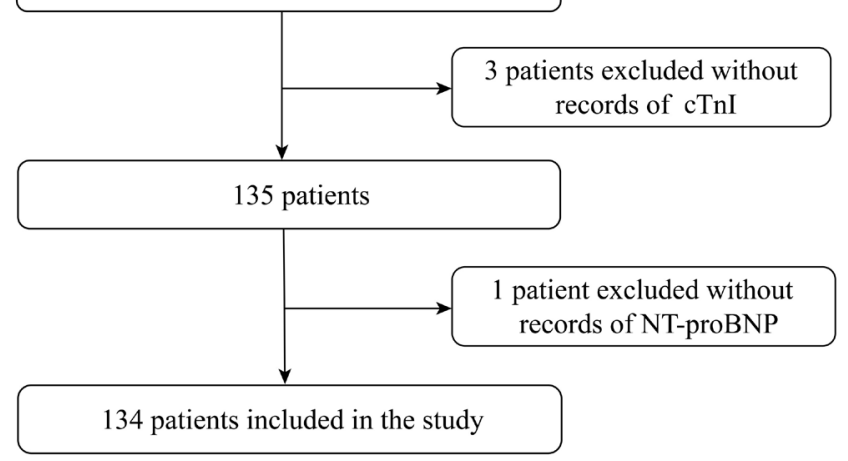

Figure 1. Flowchart of screening and enrollment of patients. COVID-19: coronavirus disease 2019; cTnl: cardiac troponin I; NT-proBNP: N-terminal pro-B-type natriuretic peptide.

guidance. ${ }^{[10]}$ Among them, three patients had no record of cTnI and one patient had no record of NT-proBNP. Thus, we enrolled 134 participants in this study. A flowchart of screening and enrollment of the patients is shown in Figure 1. The study was approved by the Ethics Committees of Peking University First Hospital (number 2020-236).

\section{Data collection}

Demographic characteristics (age and sex), symptoms, medical history, laboratory results, treatments, and clinical outcomes were collected from electronic medical records and reviewed by a team of trained physicians.

CTnI levels were measured using the chemiluminescent immunoassay method (Abbott, Chicago, USA), and NT-proBNP levels were measured using the electrical chemiluminescent immunoassay method (Roche, Basel, Switzerland). According to the test manual and reagent instructions of Tongji Hospital, the normal reference range of cTnI uses the 99th percentile of healthy people as the upper limit, which is $\leq 26.2 \mathrm{ng} / \mathrm{L}$. The normal reference range of NT-proBNP is $5.0-97.3 \mathrm{ng} / \mathrm{L}$ for $18-44$ years of age, $5.0-121.0 \mathrm{ng} / \mathrm{L}$ for $45-54$ years of age, $5.0-198.0 \mathrm{ng} / \mathrm{L}$ for 55-64 years of age, 5.0-285.0 ng/L for 65-74 years of age, and $5.0-526.0 \mathrm{ng} / \mathrm{L}$ for $\geq 75$ years of age. cTnI elevation was defined as the highest blood level of cTnI above the 99th percentile reference limit, and NT-proBNP elevation was defined as the highest blood NT-proBNP level above the normal range of related age. The estimated glomerular filtration rate (eGFR) was calculated using the Chronic Kidney Disease Epidemiology Collaboration equation. ${ }^{[1]}$

Severity of the disease was staged according to the Diagnosis and Treatment Plan for COVID-19 (Tentative, the seventh version) issued by the National Health Committee of the People's Republic of China. A severe case was defined as 
having one of the following: (1) respiratory rate $>30 /$ min, (2) pulse oxygen saturation $\left(\mathrm{SpO}_{2}\right) \leq 93 \%$, and (3) arterial oxygen pressure $\left(\mathrm{PaO}_{2}\right)$ / fraction of inspired oxygen $\left(\mathrm{FiO}_{2}\right)$ ratio $\leq 300 \mathrm{mmHg}$. A critically ill case was defined as having at least one of the following criteria: shock, respiratory failure requiring mechanical ventilation, and extrapulmonary organ failure requiring intensive care. The clinical outcomes were defined as death and ventilator use within 30 days after admission.

\section{Statistical analysis}

Data are shown as mean \pm standard deviation for continuous variables that were normally distributed, and as median and interquartile range (IQR) for continuous variables that were non-normally distributed. Categorical variables are expressed as number and proportion. Proportions were compared using the $\chi^{2}$ test. Mean values were compared using the $t$-test when the data were normally distributed and the Kruskal-Wallis test when they were not.

Multivariate Cox regression was used to determine the association of cTnI levels, NT-proBNP levels, and their combined effects on death rate. The hazard ratio (HR) and $95 \%$ confidence interval (CI) are shown to indicate the effect. Survival curve was plotted using the KaplanMeier method and compared among different groups of patients using the log-rank test. All analyses were considered statistically significant at $P<0.05$. All statistical analyses were performed with EmpowerStats (http:/ /www. empowerstats.com/en/index.html) and R-Project (https:/ / www.r-project.org/, version 3.4.3).

\section{RESULTS}

\section{Patients' characteristics and laboratory findings}

Characteristics and laboratory results of the patients are summarized in Table 1 . The mean age was $62.0 \pm 14.6$ years, and $50.8 \%(68 / 134)$ of the patients were men. Common symptoms such as fever $(116 / 134,86.6 \%)$, cough $(112 / 134,83.6 \%)$, sputumproduction $(83 / 134,62.0 \%)$, and shortness of breath $(89 / 134,66.4 \%)$ were observed in the patients. A total of $18.7 \%(25 / 134)$ of the patients experienced chest pain and $25.4 \%(34 / 134)$ had palpitation.

Mean/median levels of $\mathrm{SpO}_{2}$, lymphocytes, and eGFR were significantly lower, whereas mean/median levels of white blood cell (WBC) count, high-sensitivity C-reactive protein (hsCRP), D-dimer, cTnI levels, NT-proBNP levels, and creatinine kinase isoenzyme-MB (CK-MB) were significantly higher in patients with cTnI elevation compared with those without. In patients with elevated NTproBNP levels, mean/median levels of $\mathrm{SpO}_{2}$, lymphocytes, and eGFR were significantly lower, and mean/median levels of WBC count, hsCRP, D-dimer, cTnI, NT-proBNP, and CK-MB were significantly higher compared with patients without.

\section{Treatment and clinical outcomes}

Table 2 shows the treatments and clinical outcomes for different groups of patients. In all the included patients, $22.4 \%$ $(30 / 134)$ and $9.0 \%(12 / 134)$ of the patients received noninvasive ventilation and invasive ventilation, respectively. A total of $23.9 \%(32 / 134)$ of the patients used glucocorticoids, and $17.9 \%(24 / 134)$ received intravenous immunoglobulin therapy. A total of $9(6.7 \%)$ patients required admission to the ICU. The overall mortality rate in our study was $18.7 \%$. Patients with cTnI elevation had higher rates of glucocorticoid use $(72.0 \% v \mathrm{~s}$. $12.8 \%, P<0.001)$, noninvasive ventilator use $(80.0 \% v$ s. $9.2 \%, P<0.001)$, invasive ventilator use $(40.0 \%$ vs. $1.8 \%$, $P<0.001)$, ICU admission ( $28.0 \%$ vs. $1.8 \%, P<0.001)$, being critically ill $(84.0 \%$ vs. $11.0 \%, P<0.001)$, and death $(68.0 \%$ vs. $7.3 \%, P<0.001)$ than those with normal cTnI levels. Patients with elevated NT-proBNP levels had a significantly higher proportion of glucocorticoid use $(46.7 \%$ vs. $5.4 \%$, $P<0.001)$, noninvasive ventilator use $(45.0 \%$ vs. $4.1 \%$, $P<0.001)$, invasive ventilator use $(18.3 \%$ vs. $1.4 \%$, $P<0.001)$, ICU admission $(13.3 \%$ vs. $1.4 \%, P=0.006)$, being critically ill $(50.0 \%$ vs. $4.1 \%, P<0.001)$, and death $(40.0 \%$ vs. $1.4 \%, P<0.001)$ than those with normal NTproBNP levels.

\section{Associations of cTnI elevation and NT-proBNP elevation with death}

The associations of cTnI levels, NT-proBNP levels, and their combined effect on the mortality rate were determined by multivariate Cox regression (Table 3). We adjusted for sex, age, history of hypertension, coronary heart disease, diabetes, chronic pulmonary disease, $\mathrm{SpO}_{2}$, and eGFR in the regression model. We found that the risk of death increased by $633 \%$ (HR 7.33, 95\% CI 2.56-21.00, $P<0.001$ ) in patients with cTnI elevation compared with those without. Similarly, patients with elevated NT-proBNP levels had a greater risk of death (HR 27.88, 95\% CI 3.55-218.78, $P=0.002)$ than those with normal NT-proBNP levels in multivariate Cox regression. To detect the joint effect of cTnI and NT-proBNP elevation, we divided the patients into three groups according to the incidence of cTnI and NT-proBNP elevation: group 1, patients without cTnI or NT-proBNP elevation; group 2, patients with either cTnI or NT-proBNP elevation; and group 3, patients with both cTnI and NT-proBNP elevation. Cox regression analysis showed that compared to group 1, group 2 had a higher risk of death (HR 15.08, 95\% CI 1.82-124.99, $P=0.012$ ), and group 3 had the highest risk of death (HR 53.87, 95\% CI 6.31-459.91, $P<0.001)$. In addition, multivariate Cox regression analysis was done to detect the association of progression of cTnI and NT-proBNP levels during 


\begin{tabular}{|c|c|c|c|c|c|c|c|}
\hline \multirow[t]{2}{*}{ Variables } & \multirow[t]{2}{*}{ All $(n=134)$} & \multicolumn{2}{|l|}{ cTnl elevated } & \multirow[t]{2}{*}{$P$-value } & \multicolumn{2}{|c|}{ NT-proBNP elevated } & \multirow[t]{2}{*}{$P$-value } \\
\hline & & No $(n=109)$ & Yes $(n=25)$ & & No $(n=74)$ & Yes $(n=60)$ & \\
\hline Age, years & $62.0 \pm 14.6$ & $61.2 \pm 14.8$ & $65.5 \pm 13.8$ & 0.192 & $60.5 \pm 14.1$ & $63.9 \pm 15.2$ & 0.178 \\
\hline Male, \% & $68(50.8 \%)$ & $49(45.0 \%)$ & $19(76.0 \%)$ & 0.005 & $35(47.3 \%)$ & $33(55.0 \%)$ & 0.375 \\
\hline \multicolumn{8}{|l|}{ Signs and symptoms } \\
\hline $\mathrm{SpO}_{2}$ at admission, $\%$ & $92.2 \pm 7.3$ & $93.9 \pm 5.2$ & $85.2 \pm 10.5$ & $<0.001$ & $94.2 \pm 5.3$ & $89.8 \pm 8.6$ & $<0.001$ \\
\hline $\begin{array}{l}\text { SBP at admission, } \\
\mathrm{mmHg}\end{array}$ & $133.5 \pm 22.4$ & $133.7 \pm 22.1$ & $\begin{array}{l}132.9 \pm \\
24.2\end{array}$ & 0.874 & $131.1 \pm 18.5$ & $136.6 \pm 26.4$ & 0.158 \\
\hline $\begin{array}{l}\text { DBP at admission, } \\
\mathrm{mmHg}\end{array}$ & $82.6 \pm 14.8$ & $82.2 \pm 14.5$ & $84.4 \pm 16.4$ & 0.511 & $81.7 \pm 13.3$ & $83.8 \pm 16.6$ & 0.409 \\
\hline $\begin{array}{l}\text { Heart rate at } \\
\text { admission, bpm }\end{array}$ & $97.0 \pm 18.0$ & $95.6 \pm 15.5$ & $\begin{array}{l}102.7 \pm \\
25.8\end{array}$ & 0.078 & $95.7 \pm 15.3$ & $98.5 \pm 20.9$ & 0.373 \\
\hline Fever, \% & $116(86.6 \%)$ & $93(85.3 \%)$ & $23(92.0 \%)$ & 0.377 & $63(85.1 \%)$ & $53(88.3 \%)$ & 0.589 \\
\hline Cough, \% & $112(83.6 \%)$ & $90(82.6 \%)$ & $22(88.0 \%)$ & 0.509 & $59(79.7 \%)$ & $53(88.3 \%)$ & 0.181 \\
\hline $\begin{array}{l}\text { Sputum production, } \\
\%\end{array}$ & $83(62.0 \%)$ & $65(60.0 \%)$ & $18(72.0 \%)$ & 0.251 & $41(55.4 \%)$ & $42(70.0 \%)$ & 0.084 \\
\hline $\begin{array}{l}\text { Shortness of breath, } \\
\%\end{array}$ & $89(66.4 \%)$ & $71(65.1 \%)$ & $18(72.0 \%)$ & 0.512 & $44(59.5 \%)$ & $45(75.0 \%)$ & 0.058 \\
\hline Chest pain, \% & $25(18.7 \%)$ & $20(18.3 \%)$ & $5(20.0 \%)$ & 0.848 & $11(14.9 \%)$ & $14(23.3 \%)$ & 0.211 \\
\hline Palpitation, \% & $34(25.4 \%)$ & $30(27.5 \%)$ & $4(16.0 \%)$ & 0.232 & $18(24.3 \%)$ & $16(26.7 \%)$ & 0.757 \\
\hline Abdominal pain, $\%$ & $25(18.7 \%)$ & $23(21.1 \%)$ & $2(8.0 \%)$ & 0.129 & $13(17.6 \%)$ & $12(20.0 \%)$ & 0.719 \\
\hline Fatigue, $\%$ & $82(61.2 \%)$ & $69(63.3 \%)$ & $13(52.0 \%)$ & 0.296 & $46(62.2 \%)$ & $36(60.0 \%)$ & 0.798 \\
\hline \multicolumn{8}{|l|}{ Medical history } \\
\hline $\begin{array}{l}\text { Coronary heart } \\
\text { disease, \% }\end{array}$ & $26(19.4 \%)$ & $21(19.3 \%)$ & $5(20.0 \%)$ & 0.933 & $14(18.9 \%)$ & $12(20.0 \%)$ & 0.875 \\
\hline Hypertension, \% & $62(46.3 \%)$ & $50(45.9 \%)$ & 12 (48.0\%) & 0.847 & $30(40.5 \%)$ & $32(53.3 \%)$ & 0.140 \\
\hline $\begin{array}{l}\text { Chronic pulmonary } \\
\text { disease, \% }\end{array}$ & $21(15.7 \%)$ & $15(13.8 \%)$ & $6(24.0 \%)$ & 0.204 & $10(13.5 \%)$ & $11(18.3 \%)$ & 0.445 \\
\hline $\begin{array}{l}\text { Chronic kidney } \\
\text { disease, \% }\end{array}$ & $9(6.7 \%)$ & $5(4.6 \%)$ & $4(16.0 \%)$ & 0.040 & $2(2.7 \%)$ & $7(11.7 \%)$ & 0.039 \\
\hline Diabetes, \% & $27(20.1 \%)$ & $20(18.4 \%)$ & $7(28.0 \%)$ & 0.278 & $15(20.3 \%)$ & $12(20.0 \%)$ & 0.969 \\
\hline \multicolumn{8}{|l|}{ Laboratory results } \\
\hline WBC, $\times 10^{9} / \mathrm{L}$ & $5.5(4.4-7.7)$ & $5.2(4.3-6.6)$ & $\begin{array}{l}8.5(6.8- \\
13.33)\end{array}$ & $<0.001$ & $5.0(4.2-5.8)$ & $7.2(5.0-10.6)$ & $<0.001$ \\
\hline $\begin{array}{l}\text { Lymphocytes, } \\
\times 10^{9} / \mathrm{L}\end{array}$ & $0.9(0.6-1.4)$ & $1.0(0.7-1.4)$ & $0.6(0.5-1.1)$ & 0.010 & $1.1(0.8-1.6)$ & $0.8(0.5-1.1)$ & $<0.001$ \\
\hline Hemoglobin, g/L & $124.1 \pm 20.7$ & $122.6 \pm 16.8$ & $\begin{array}{l}130.7 \pm \\
32.4\end{array}$ & 0.077 & $125.3 \pm 12.9$ & $122.7 \pm 27.4$ & 0.466 \\
\hline Platelets, $\times 10^{9} / \mathrm{L}$ & $\begin{array}{l}231.5 \\
(159.3-291.8)\end{array}$ & $\begin{array}{l}233.0(165.0- \\
291.0)\end{array}$ & $\begin{array}{l}222.0 \\
(138.0- \\
295.0)\end{array}$ & 0.673 & $\begin{array}{l}241.0 \\
(168.0- \\
291.0)\end{array}$ & $\begin{array}{l}216.0 \\
(147.0-292.8)\end{array}$ & 0.390 \\
\hline Creatinine, $\mu \mathrm{mol} / \mathrm{L}$ & $\begin{array}{l}72.5 \\
(58.0-91.0)\end{array}$ & $70.0(57.0-87.0)$ & $\begin{array}{l}91.0 \\
(69.0-105.0)\end{array}$ & 0.003 & $\begin{array}{l}65.0 \\
(55.0-1.8)\end{array}$ & $\begin{array}{l}85.5 \\
(66.0-99.0)\end{array}$ & $<0.001$ \\
\hline eGFR, $\mathrm{ml} / \mathrm{min} / 1.73^{\mathrm{m} 2}$ & $\begin{array}{l}78.2 \\
(57.1-94.2)\end{array}$ & $82.3(60.0-95.2)$ & $\begin{array}{l}59.6 \\
(46.8-78.3)\end{array}$ & 0.003 & $\begin{array}{l}85.5 \\
(71.2-99.7)\end{array}$ & $\begin{array}{l}61.1 \\
(49.6-84.4)\end{array}$ & $<0.001$ \\
\hline $\mathrm{hsCRP}, \mathrm{mg} / \mathrm{L}$ & $\begin{array}{l}33.1 \\
(6.2-81.0)\end{array}$ & $26.1(5.3-57.9)$ & $\begin{array}{l}121.4 \\
(40.2-210.1)\end{array}$ & $<0.001$ & $\begin{array}{l}11.9 \\
(3.5-40.6)\end{array}$ & $\begin{array}{l}59.6 \\
(27.2-142.6)\end{array}$ & $<0.001$ \\
\hline Procalcitonin, ng/mL & $\begin{array}{l}0.05 \\
(0.03-0.14)\end{array}$ & $0.04(0.03-0.10)$ & $\begin{array}{l}0.32 \\
(0.12-0.57)\end{array}$ & $<0.001$ & $\begin{array}{l}0.03 \\
(0.02-0.08)\end{array}$ & $\begin{array}{l}0.12(0.05- \\
0.36)\end{array}$ & $<0.001$ \\
\hline D-Dimer, $\mu \mathrm{g} / \mathrm{mL}$ & $1.2(0.5-2.3)$ & $1.0(0.5-2.0)$ & $\begin{array}{l}2.6 \\
(1.4-16.2)\end{array}$ & $<0.001$ & $\begin{array}{l}0.7 \\
(0.4-1.8)\end{array}$ & $1.7(0.9-2.8)$ & $<0.001$ \\
\hline $\mathrm{cTnl}, \mathrm{pg} / \mathrm{mL}$ & $\begin{array}{l}4.6 \\
(2.2-10.3)\end{array}$ & $3.6(1.9-7.2)$ & $\begin{array}{l}57.6 \\
(11.2-234.8)\end{array}$ & $<0.001$ & $\begin{array}{l}3.1 \\
(1.9-5.4)\end{array}$ & $9.4(4.1-26.4)$ & $<0.001$ \\
\hline $\begin{array}{l}\text { Myohemoglobin, } \\
\mu \mathrm{g} / \mathrm{L}\end{array}$ & $\begin{array}{l}58.6 \\
(37.8-129.1)\end{array}$ & $53.0(35.5-90.9)$ & $\begin{array}{l}131.7 \\
(60.5-247.4)\end{array}$ & $<0.001$ & $\begin{array}{l}43.0(32.0- \\
73.1)\end{array}$ & $\begin{array}{l}119.8 \\
(52.8-175.1)\end{array}$ & $<0.001$ \\
\hline CK-MB, ng/mL & $0.9(0.4-1.7)$ & $0.7(0.4-1.4)$ & $1.7(1.1-5.4)$ & $<0.001$ & $0.7(0.4-1.3)$ & $1.3(0.6-2.4)$ & $<0.001$ \\
\hline NT pro-BNP, pg/mL & $\begin{array}{l}180.0 \\
(67.0-456.5)\end{array}$ & $142.0(64.0-292.0)$ & $\begin{array}{l}852.0 \\
(386.0- \\
1658.0)\end{array}$ & $<0.001$ & $\begin{array}{l}92.0(56.8- \\
179.8)\end{array}$ & $\begin{array}{l}523.5 \\
(291.5- \\
1011.8)\end{array}$ & $<0.001$ \\
\hline
\end{tabular}

$\mathrm{SpO}_{2}$ : pulse oxygen saturation; SBP: systolic blood pressure; DBP: diastolic blood pressure; WBC: white blood cell; eGFR: estimated glomerular filtration rate; hsCRP: high-sensitivity C-reactive protein; cTnl: cardiac troponin I; CK-MB: creatinine kinase isoenzyme-MB; NT-proBNP: N-terminal pro-B-type natriuretic peptide. 
Weng et al.: Predictive value of cTnI and NT-proBNP in COVID-19 patients

\begin{tabular}{|c|c|c|c|c|c|c|c|}
\hline \multirow[t]{2}{*}{ Variable } & \multirow[t]{2}{*}{$n(\%)$} & \multicolumn{2}{|l|}{ cTnl elevated } & \multirow[t]{2}{*}{$P$-value } & \multicolumn{2}{|c|}{ NT-proBNP elevated } & \multirow[t]{2}{*}{$P$-value } \\
\hline & & No $(n=109)$ & Yes $(n=25)$ & & No $(n=74)$ & Yes $(n=60)$ & \\
\hline \multicolumn{8}{|l|}{ Treatment } \\
\hline Antiviral treatment, \% & $115(85.8 \%)$ & $95(87.1 \%)$ & $20(80.0 \%)$ & 0.355 & $61(82.4 \%)$ & $54(90.0 \%)$ & 0.212 \\
\hline Antibiotic treatment, \% & $63(47.0 \%)$ & $40(36.7 \%)$ & $23(92.0 \%)$ & $<0.001$ & $21(28.4 \%)$ & $42(70.0 \%)$ & $<0.001$ \\
\hline $\begin{array}{l}\text { Intravenous immunoglobulin } \\
\text { therapy, \% }\end{array}$ & $24(17.9 \%)$ & $16(14.7 \%)$ & $8(32.0 \%)$ & 0.042 & $6(8.1 \%)$ & $18(30.0 \%)$ & 0.001 \\
\hline Glucocorticoids, \% & $32(23.9 \%)$ & $14(12.8 \%)$ & $18(72.0 \%)$ & $<0.001$ & $4(5.4 \%)$ & $28(46.7 \%)$ & $<0.001$ \\
\hline Noninvasive ventilation, \% & $30(22.4 \%)$ & $10(9.2 \%)$ & $20(80.0 \%)$ & $<0.001$ & $3(4.1 \%)$ & $27(45.0 \%)$ & $<0.001$ \\
\hline $\begin{array}{l}\text { Invasive mechanical } \\
\text { ventilation, \% }\end{array}$ & $12(9.0 \%)$ & $2(1.8 \%)$ & $10(40.0 \%)$ & $<0.001$ & $1(1.4 \%)$ & $11(18.3 \%)$ & $<0.001$ \\
\hline \multicolumn{8}{|l|}{ Clinical outcomes } \\
\hline ICU admission, \% & $9(6.7 \%)$ & $2(1.8 \%)$ & $7(28.0 \%)$ & $<0.001$ & $1(1.4 \%)$ & $8(13.3 \%)$ & 0.006 \\
\hline Critically ill, \% & $33(24.6 \%)$ & $12(11.0 \%)$ & $21(84.0 \%)$ & $<0.001$ & $3(4.1 \%)$ & $30(50.0 \%)$ & $<0.001$ \\
\hline Death, \% & $25(18.7 \%)$ & $8(7.3 \%)$ & $17(68.0 \%)$ & $<0.001$ & $1(1.4 \%)$ & $24(40.0 \%)$ & $<0.001$ \\
\hline Ventilator use, \% & $31(23.1 \%)$ & $10(9.2 \%)$ & $21(84.0 \%)$ & $<0.001$ & $3(4.1 \%)$ & $28(46.7 \%)$ & $<0.001$ \\
\hline
\end{tabular}

cTnl: cardiac troponin I; NT-proBNP: N-terminal pro-B-type natriuretic peptide; ICU: intensive care unit.

\begin{tabular}{|c|c|c|c|c|c|c|}
\hline \multicolumn{7}{|l|}{ Death } \\
\hline \multirow[t]{2}{*}{ Variables } & \multicolumn{4}{|c|}{ Unadjusted } & \multicolumn{2}{|l|}{ Adjusted* } \\
\hline & $n(\%)$ & Death $(\%)$ & $\mathrm{HR}(95 \% \mathrm{Cl})$ & $P$-value & HR (95\% Cl) & $P$-value \\
\hline Elevated cTnl levels & $25(18.7 \%)$ & $17(68.0 \%)$ & $12.94(5.55-30.18)$ & $<0.001$ & $7.33(2.56-21.00)$ & $<0.001$ \\
\hline Elevated NT-proBNP levels & $60(44.8 \%)$ & $24(40.0 \%)$ & 36.62 (4.95-270.90) & $<0.001$ & $27.88(3.55-218.78)$ & 0.002 \\
\hline \multicolumn{7}{|l|}{ Combined effect $^{\dagger}$} \\
\hline Group 1 & $73(54.5 \%)$ & $1(1.4 \%)$ & Reference & & Reference & \\
\hline Group 2 & $37(27.6 \%)$ & $7(18.9 \%)$ & $15.55(1.91-126.45)$ & 0.010 & $15.08(1.82,124.99)$ & 0.012 \\
\hline Group 3 & $24(17.9 \%)$ & $17(70.8 \%)$ & $77.08(10.22-581.35)$ & $<0.001$ & $53.87(6.31,459.91)$ & $<0.001$ \\
\hline$P$ for trend & & & & $<0.001$ & & $<0.001$ \\
\hline
\end{tabular}

${ }^{*}$ Adjusted for gender, age, history of hypertension, coronary heart disease, diabetes, chronic pulmonary disease, pulse oxygen saturation, estimated glomerular filtration rate. ${ }^{\dagger}$ Group 1 stands for patients without elevated levels of cTnl or NT-proBNP. Group 2 stands for patients with elevated levels of cTnl or NT-proBNP. Group 3 stands for patients with elevated levels of cTnl and NT-proBNP. HR: hazard ratio; Cl: confidence interval; cTnl: cardiac troponin I; NTproBNP: N-terminal pro-B-type natriuretic peptide.

hospitalization with death (Table 4). Compared to patients with normal cTnI levels during the whole hospital stay, the risk of death was the highest (HR 12.70, 95\% CI 3.94-40.88, $P<0.001)$ in patients with normal cTnI levels at admission but developed cTnI elevation during hospitalization, after adjusting for sex, age, history of hypertension, coronary heart disease, diabetes, chronic pulmonary disease, $\mathrm{SpO}_{2}$, and eGFR. Similarly, compared to patients with normal NT-proBNP levels during the whole hospital stay, the risk of death was the highest (HR 51.09, 95\% CI 5.82-448.26, $P<0.001)$ in patients with normal NT-proBNP levels at admission but developed NT-proBNP elevation during hospitalization, after adjusting for sex, age, history of hypertension, coronary heart disease, diabetes, chronic pulmonary disease, $\mathrm{SpO}_{2}$, and eGFR.
Kaplan-Meier curves (Figure 2) show that the rates of mortality increased in patients with cTnI and NT-proBNP elevation.

\section{DISCUSSION}

In this single-center retrospective study, we evaluated the predictive values of cTnI and NT-proBNP on mortality in 134 COVID-19 patients. The overall death rate was $18.7 \%$ (25 out of 134) in our study population. Both elevated levels of cTnI and NT-proBNP during hospital stay were significantly associated with an increased risk of death, after adjusting for gender, age, history of hypertension, coronary heart disease, diabetes, chronic pulmonary disease, $\mathrm{SpO}_{2}$, and eGFR. Moreover, for the first time, we found that cTnI 
Table 4: Multivariate Cox regression analysis on the association of progression of cTnl and NT-proBNP levels during hospitalization with death or ventilator use in COVID-19 patients

Death

\begin{tabular}{|c|c|c|c|c|c|c|}
\hline \multirow[t]{2}{*}{ Variables } & \multirow[b]{2}{*}{$n(\%)$} & \multirow[b]{2}{*}{ Death (\%) } & \multicolumn{2}{|l|}{ Unadjusted } & \multicolumn{2}{|l|}{ Adjusted* } \\
\hline & & & HR (95\% Cl) & $P$-value & HR (95\% Cl) & $P$-value \\
\hline \multicolumn{7}{|c|}{$\begin{array}{l}\text { Progression of CTnl levels in } \\
\text { hospitalization }^{\dagger}\end{array}$} \\
\hline Group 1 & $109(81.3 \%)$ & $8(7.3 \%)$ & Reference & & Reference & \\
\hline Group 2 & $16(11.9 \%)$ & $9(56.3 \%)$ & 10.39 (3.99-27.07) & $<0.001$ & $4.38(1.25-15.33)$ & 0.021 \\
\hline Group 3 & $9(6.7 \%)$ & $8(88.9 \%)$ & $17.96(6.69-48.25)$ & $<0.001$ & $12.70(3.94-40.88)$ & $<0.001$ \\
\hline$P$ for trend & & & & $<0.001$ & & $<0.001$ \\
\hline \multicolumn{7}{|c|}{$\begin{array}{l}\text { Progression of NT-proBNP } \\
\text { levels in hospitalization }^{\dagger}\end{array}$} \\
\hline Group 1 & $74(55.2 \%)$ & $1(1.4 \%)$ & Reference & & Reference & \\
\hline Group 2 & $48(35.8 \%)$ & $18(37.5 \%)$ & $34.03(4.54-255.15)$ & $<0.001$ & $21.51(2.63-175.74)$ & 0.013 \\
\hline Group 3 & $12(9.0 \%)$ & $6(50.0 \%)$ & $47.38(5.70-393.82)$ & $<0.001$ & $51.09(5.82-448.26)$ & $<0.001$ \\
\hline$P$ for trend & & & & $<0.001$ & & $<0.001$ \\
\hline
\end{tabular}

*Adjusted for gender, age, history of hypertension, coronary heart disease, diabetes, chronic pulmonary disease, pulse oxygen saturation, estimated glomerular filtration rate. ${ }^{\dagger}$ Group 1 stands for patients without elevated levels of cTnl or NT-proBNP. Group 2 stands for patients with elevated levels of cTnl or NT-proBNP. Group 3 stands for patients with elevated levels of cTnl and NT-proBNP. HR: hazard ratio; Cl: confidence interval; cTnl: cardiac troponin I; NT-proBNP: N-terminal pro-B-type natriuretic peptide.

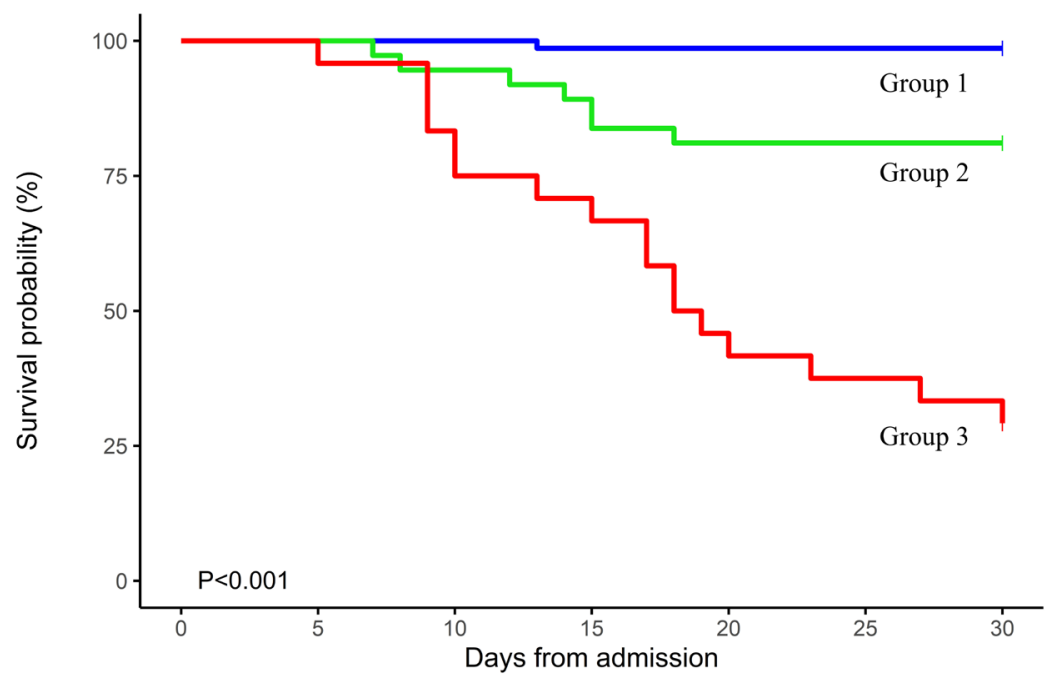

Number at risk

\begin{tabular}{|c|c|c|c|c|c|c|c|}
\hline Group 1 & 73 & 73 & 73 & 72 & 72 & 72 & 72 \\
\hline 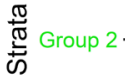 & 37 & 37 & 35 & 33 & 30 & 30 & 30 \\
\hline \multirow[t]{2}{*}{ Group 3} & 24 & 24 & 20 & 17 & 11 & 9 & 8 \\
\hline & 0 & 5 & & 15 & 20 & 25 & 30 \\
\hline
\end{tabular}

Figure 2: Kaplan-Meier curves of mortality according to the combined effect of elevated cTnl and NT-proBNP levels. Group 1 stands for patients without elevated levels of cTnl or NT-proBNP. Group 2 stands for patients with elevated levels of cTnl or NT-proBNP. Group 3 stands for patients with elevated levels of cTnl and NT-proBNP. cTnl: cardiac troponin I; NT-proBNP: N-terminal pro-B-type natriuretic peptide. 
and NT-proBNP had a joint effect on predicting death, and the progression of the two cardiac injury parameters could also predict death.

Prior to our study, several studies, including a meta-analysis, had addressed the predictive value of cTnI and NTproBNP. ${ }^{[6,9,12-14]}$ But based on those reported results, it is inconclusive whether the levels of cardiac injury parameters at admission were independently associated with death. One limitation of these studies is that most of them adjusted limited number of important covariates that could also influence the patients' outcomes. So, in our study, we included as much information as we were able to obtain, including age, gender, history of hypertension, coronary heart disease, diabetes, chronic pulmonary disease, $\mathrm{SpO}_{2}$, and eGFR. After adjusting these important covariates, we found a very significant association between the two cardiac injury parameters and death in our study population.

To our knowledge, we are the first to show the joint predictive value of elevated cTnI and NT-proBNP levels on mortality in COVID-19 patients. A previous study showed that the combination of NT-proBNP and troponin I levels had higher prognostic utility in older patients with severe sepsis or septic shock in the ICU. ${ }^{[15]}$ In our study, we found that the patients with both elevated cTnI and NT-proBNP levels had the highest risk of death. cTnI is a sensitive marker of cardiac injury, whereas NT-proBNP is a marker of heart insufficiency. Therefore, patients with elevated levels of both cTnI and NT-proBNP may have cardiac injury and dysfunction of the left and/or right ventricle. For these patients, the clinical prognosis may be worse because of severe cardiac injury and either poor or decompensated heart function after COVID-19 infection. These patients might be vulnerable to the excessive volume load during fluid resuscitation and would be less sensitive to vasopressors, which are the two vital treatments for sepsis shock caused by severe acute respiratory syndrome.

Another important contribution of our study is that we investigated the change of cardiac injury parameters and their effect in predicting death. Given the rapid deterioration of the disease in some patients, ${ }^{[16]}$ baseline levels of cardiac injury may not be an optimal marker to predict future events. In the current study, we investigated the progression trend of these two parameters and found it to be independently associated with an increased risk of death. What can be translated from our findings to clinical use is that physicians need to monitor these parameters closely, and once their levels go up, they should consider more active therapy.

These findings are important for physicians to identify a potentially high-risk patient with COVID-19 in the early stage of this disease. Close monitoring of patients with elevated levels of cTnI and NT-proBNP may be required, which is crucial to reduce case fatality rate.

Mechanisms of cardiac injury are not fully understood. Several pathophysiological pathways have been proposed to explain the elevated parameters. Myocarditis might be an explanation of the cardiac injury through direct or indirect mechanisms. ${ }^{[17]}$ Multiple autopsy findings showed the infiltration of macrophages and $\mathrm{CD}^{+} \mathrm{T}$ lymphocytes in the myocardium of COVID-19 patients, ${ }^{[18-20]}$ which was thought to be the outcome of the cytokine release syndrome. ${ }^{[21]}$ Another case report demonstrated the presence of SARS-CoV-2 viral particles in cardiac macrophages, suggesting that cardiomyocytes can be directly infected by the virus. ${ }^{[2]}$ Cardiac MRI showed that patients with severe COVID-19 had myocardial inflammation, edema, and/or diffuse myocardial fibrosis, which confirmed the incidence of myocarditis. ${ }^{[23,24]}$ Another theory for the cardiac injury was microthrombi induced by endothelial injury. ${ }^{[25]}$ Large thrombi, microangiopathy, and signs of disseminated intravascular coagulation were observed in severe cases of COVID-19. ${ }^{[26]}$ A recent study showed that the most common pathological cause of myocyte necrosis is microthrombi. ${ }^{[2]}$ Other explanations were type 1 and type 2 myocardial infarction. Inflammatory responses can directly affect atherosclerotic plaques and increase procoagulant and prothrombotic activity, ${ }^{[28]}$ which could result in the acute coronary syndrome. ${ }^{[29]}$ Oxygen supply-demand mismatch in severe and critically ill COVID-19 patients with hypoxia, acidosis, or hypotension can explain the type 2 myocardial infarction. ${ }^{[29]}$

\section{LIMITATIONS}

The major limitation of this study is that it is a retrospective study, and because of the small sample size and lack of some key clinical examinations, like ECG/echocardiogram, it was difficult to explore the underlying mechanism. Further large-scale prospective studies are warranted to confirm our discovery.

\section{CONCLUSIONS}

In conclusion, elevated cTnI and NT-proBNP levels have a joint effect in predicting death in COVID-19 patients. The progression of these two parameters needs to be monitored closely, since they could also predict death in these patients.

\section{Source of Funding}

This research was supported by Youth Clinical Research Project of Peking University First Hospital (2018CR05). 


\section{Conflict of Interests}

None.

\section{Acknowledgments}

We are grateful to the frontline medical workers in Wuhan and to all the patients involved in this study. We thank Dr. Xinmin Liu, who was the leader of our medical team, for the support of this study. We thank Professor Li Yang from the Department of Nephology in Peking University First Hospital for the help of directing the construction of the database.

\section{Authors Contributions}

Haoyu Weng, Haichao Li, Yan Zhang, and Jianping Li designed the study. Fan Yang, Han Jin, Long Zhang, Shengcong Liu, Hongyu Yang, and Xizi Zheng were responsible for collecting data and establishing the database. Fangfang Fan, Zhihao Liu, Yuxi Li, and Tieci Yi did the data analysis. Haoyu Weng and Jianping Li wrote and revised the manuscript. All the authors approved the final version of the manuscript for publication. The manuscript has been read and approved by all the authors, and each author believes that the manuscript represents honest work.

\section{REFERENCE}

1. Jin Y, Yang H, Ji W, Wu W, Chen S, Zhang W, et al. Virology, Epidemiology, Pathogenesis, and Control of COVID-19. Viruses 2020; 12: 372.

2. Zhou F, Yu T, Du R, Fan G, Liu Y, Liu Z, et al. Clinical course and risk factors for mortality of adult inpatients with COVID-19 in Wuhan, China: a retrospective cohort study. Lancet 2020; 395:1054-62.

3. Wu Z, McGoogan JM. Characteristics of and Important Lessons From the Coronavirus Disease 2019 (COVID-19) Outbreak in China: Summary of a Report of 72314 Cases From the Chinese Center for Disease Control and Prevention. JAMA 2020; 323:1239-42.

4. Wang D, Hu B, Hu C, Zhu F, Liu X, Zhang J, et al. Clinical Characteristics of 138 Hospitalized Patients With 2019 Novel Coronavirus-Infected Pneumonia in Wuhan, China. JAMA 2020; 323:1061-9.

5. Akhmerov A, Marbán E. COVID-19 and the Heart. Circ Res 2020; 126 : 1443-55.

6. Shi S, Qin M, Shen B, Cai Y, Liu T, Yang F, et al. Association of Cardiac Injury With Mortality in Hospitalized Patients With COVID-19 in
Wuhan, China. JAMA Cardiol 2020; 5:802-10.

7. Metkus TS, Sokoll LJ, Barth AS, Czarny MJ, Hays AG, Lowenstein CJ, et al. Myocardial Injury in Severe COVID-19 Compared With NonCOVID-19 Acute Respiratory Distress Syndrome. Circulation 2021; 143: 553-65.

8. Guo T, Fan Y, Chen M, Wu X, Zhang L, He T, et al. Cardiovascular Implications of Fatal Outcomes of Patients With Coronavirus Disease 2019 (COVID-19). JAMA Cardiol 2020; 5: 811-8.

9. Gao L, Jiang D, Wen XS, Cheng XC, Sun M, He B, et al. Prognostic value of NT-proBNP in patients with severe COVID-19. Respir Res 2020; 21: 83.

10. Organization WH. Clinical management of severe acute respiratory infection when novel coronavirus ( $\mathrm{nCoV}$ ) infection is suspected, Interim guidance. Available from: https://www.who.int/docs/ default-source/coronaviruse/clinical-management-of-novel-cov. pdf?sfvrsn=bc7da517_2. Accessed on Mar. 13, 2020.

11. Levey AS, Stevens LA, Schmid CH, Zhang YL, Castro AF, 3rd, Feldman $\mathrm{HI}$, et al. A new equation to estimate glomerular filtration rate. Ann Intern Med 2009; 150: 604-12.

12. Li JW, Han TW, Woodward M, Anderson CS, Zhou H, Chen YD, et al. The impact of 2019 novel coronavirus on heart injury: A systemic review and Meta-analysis. Prog Cardiovasc Dis 2020; 63:518-24.

13. Ghio S, Baldi E, Vicentini A, Lenti MV, Di Sabatino A, Di Matteo A, et al. Cardiac involvement at presentation in patients hospitalized with COVID-19 and their outcome in a tertiary referral hospital in Northern Italy. Intern Emerg Med 2020; 15: 1457-65.

14. Maeda T, Obata R, Rizk D, Kuno T. Cardiac Injury and Outcomes of Patients with COVID-19 in New York City. Heart Lung Circ 2021;30: 848-53.

15. Cheng H, Fan WZ, Wang SC, Liu ZH, Zang HL, Wang LZ, et al. $\mathrm{N}$-terminal pro-brain natriuretic peptide and cardiac troponin I for the prognostic utility in elderly patients with severe sepsis or septic shock in intensive care unit: A retrospective study. J Crit Care 2015; 30: 654. e9-14.

16. Goh KJ, Choong MC, Cheong EH, Kalimuddin S, Duu Wen S, Phua GC, et al. Rapid Progression to Acute Respiratory Distress Syndrome: Review of Current Understanding of Critical Illness from COVID-19 Infection. Ann Acad Med Singapore 2020; 49: 108-18.

17. Unudurthi SD, Luthra P, Bose RJC, McCarthy JR, Kontaridis MI. Cardiac inflammation in COVID-19: Lessons from heart failure. Life Sci 2020; 260: 118482.

18. Wichmann D, Sperhake JP, Lütgehetmann M, Steurer S, Edler C, Heinemann A, et al. Autopsy Findings and Venous Thromboembolism in Patients With COVID-19: A Prospective Cohort Study. Ann Intern Med.42 2020; 173: 268-77.

19. Xu Z, Shi L, Wang Y, Zhang J, Huang L, Zhang C, et al Pathological findings of COVID-19 associated with acute respiratory distress syndrome. Lancet Respir Med 2020; 8:420-2.

20. Sala S, Peretto G, Gramegna M, Palmisano A, Villatore A, Vignale D, et al. Acute myocarditis presenting as a reverse Tako-Tsubo syndrome in a patient with SARS-CoV-2 respiratory infection. Eur Heart J 2020; 41: $1861-2$.

How to cite this article: Weng $\mathrm{H}$, Yang $\mathrm{F}$, Zhang L, Jin $\mathrm{H}$, Liu S, Fan $\mathrm{F}$, et al. Joint Predictive Value of cTnl and NT-proBNP on Mortality in COVID-19 Patients: A Retrospective Research in Wuhan, China. J Transl Intern Med 2021; 9: 177-84. 